\title{
¿Racionalidad científico-instrumental en el derecho? \\ Los límites de la universalidad del conocimiento jurídico*
}

\author{
Anderson Vichinkeski Teixeira**
}

\begin{abstract}
Resumen:
En este ensayo se propone, en primer lugar, analizar el tema de la relevancia de la racionalidad científico-instrumental en las ciencias en general y la dificultad de su utilización en la ciencia jurídica. Aunque en este momento se tenga presente la idea de que el derecho es una ciencia, hay otras concepciones posibles sobre qué racionalidad debe ubicar a la producción jurídica, en contraste con la idea típica de ciencia como racionalidad instrumental. Posteriormente, serán examinadas las dificultades que surgieron a la raíz del proceso de evaluación editorial de investigaciones recibidas en revistas jurídicas, en especial en cuanto a las remitidas para las grandes bases de datos e índices internacionales, ya que las diferentes tradiciones jurídicas implican diferentes formas de racionalidad en la construcción del pensamiento. Algunos datos extraídos de la revista RECHTD, entre otras, en las cuales el autor actúa como editor o como revisor en el comité de redacción, serán apuntados a modo de respaldar las posibles hipótesis aquí señaladas.
\end{abstract}

Palabras clave: racionalidad jurídica, investigación jurídica, revistas científicas.

\section{Scientific-instrumental rationality in Law? The limits of the universality of legal knowledge}

\begin{abstract}
:
In this essay it is proposed, in the first place, to analyze the issue of the relevance of scientific-instrumental rationality in science in general and the difficulty of its use in legal science. Although the idea is kept in mind that Law is a science, there are other possible conceptions about what rationality should locate legal production, in contrast to the typical idea of science as instrumental rationality. Subsequently, the difficulties that arose as a result of the process of editorial evaluation of researches received in legal journals will be examined, especially in regard to those submitted for large international databases and indexes, since the different legal traditions imply different forms of rationality in the construction of thought. Some data extracted from the RECHTD journal, among others, in which the author acts as editor and/or as member in the editorial committee, will be pointed out in order to support the possible hypotheses indicated here.
\end{abstract}

Keywords: legal rationality, legal research, scientific journals.

\footnotetext{
* Artículo de investigación Número Especial de Aniversario. Recibido el 26 de marzo de 2020 y aceptado para su publicación el 11 deagosto de 2020.

** Profesor en la Universidade do Vale do Rio dos Sinos, Brasil. / andersonvt@unisinos.br / orcid. org/ 0000-0001-7085-0375
} 


\section{Sumario}

1. Consideraciones introductorias 2. Racionalidades diferentes, ciencias diferentes: ¿cómo evaluar con los mismos estándares editoriales? 3. Los límites de la racionalidad científico-instrumental en derecho y la leyenda de su uso universal 4. Algunas consideraciones finales basadas en experiencias de la RECHTD y otras revistas 5 . Referencias

\section{Consideraciones introductorias}

La racionalidad científico-instrumental se ha consolidado, para las ciencias en general, como un proceso racional cuya universalidad de los resultados de la investigación pueden desarrollarse en los contextos más variopintos posibles, independientemente de dónde se ubique el investigador. En áreas como las ciencias médicas y las ciencias exactas, la existencia de condiciones intrínsecamente validadas permite que el mismo experimento se lleve a cabo y se repita varias veces, sin importar el lugar donde esté el investigador. Básicamente, todo se debe a la existencia de principios rectores comunes y métodos compartidos de análisis vinculados con los objetos de estudio.

Sin embargo, en el derecho, dada su pretensión de ser una verdadera "ciencia jurídica", la racionalidad científico-instrumental tiene una aplicabilidad extremadamente limitada no solo para el derecho, sino también para las ciencias sociales, como señaló Max Horkheimer cuando destacó que había diferencias sustanciales entre la razón instrumental y la razón crítica. ${ }^{1}$ La idea misma de que el derecho es una ciencia se muestra como un elemento discutible, ya que diversas posibles concepciones de racionalidad pueden afectar la producción jurídica.

Por tanto, se harán algunas breves consideraciones sobre el problema del método en la investigación jurídica y, en seguida, serán apuntadas las dificultades que surgieron en el proceso de evaluación editorial de investigaciones recibidas en revistas jurídicas incluidas en grandes bases de datos y con indexadores internacionales. Finalmente, será examinado cómo las diferentes tradiciones jurídicas en las que se encuentran los autores de los artículos terminan imbricando decisivamente las diferentes formas de racionalidad que los conducen a la construcción del pensamiento y, en consecuencia, a los resultados finales de sus investigaciones. Nuestras

1 Véase: Horkheimer, Max, Eclipse de la raison, Paris, Payot, 1974, pp. 24-27. 
experiencias personales se aportarán especialmente a partir la gestión editorial de la revista RECHTD, entre otras en las cuales el autor de este ensayo actúa como colaborador, ya que tiene por objetivo defender la hipótesis de que la definición de un perfil editorial delimitado metodológicamente no puede menospreciar las investigaciones que se originan con bases en diferentes tradiciones jurídicas.

\section{Racionalidades diferentes, ciencias diferentes: ¿cómo evaluar desde los mismos estándares editoriales?}

Aunque pueda mencionarse como un aspecto obvio, una reflexión sobre racionalidades presupone la existencia de una definición preliminar sobre el método y el modo en que se encaja en el nivel epistemológico. Además, las diferentes orientaciones filosóficas de los estudios en dichas epistemologías no deben crear imprecisiones teóricas, sino que deben servir como fuentes capaces de brindar una precisa delimitación del enfoque de la investigación.

Así, considerando las distinciones existentes en el ámbito de la filosofía de la ciencia, tres son los enfoques de investigación notoriamente reconocidos: descriptivo, explicativo y normativo. ${ }^{2}$ El primero trata de describir, con mayor grado de concretización, las relaciones concernientes a los objetos de observación de esa determinada ciencia; el segundo trata de presentar hipótesis para explicar las relaciones causales que constituyen el objeto de investigación; el tercero presenta proposiciones prescriptivas respecto a valorar en qué medida se ajusta la ciencia a los conceptos universales de determinado conocimiento científico. Por cierto, la noción y relevancia de un método será decisivamente diferente según cada uno de esos tres enfoques.

Karl Popper, reconocido por sus trabajos sobre la filosofía de la ciencia, fue un epistemólogo con notable orientación normativista que puso de relieve la existencia de elementos factibles en condiciones de proporcionar una mayor solidez para la ciencia cuando esta enfrenta los problemas del conocimiento. Tales problemas pueden considerarse a partir de dos clases diferentes de enunciados: (1) universales, como hipótesis que tienen el carácter de leyes universales; y (2) singulares, como descripciones de acontecimientos

\footnotetext{
${ }^{2}$ Sobre el tema, véase: Bunge, Mario, La ciencia: su método y su filosofía, Buenos Aires, Editorial Sudamericana, 1995.
} 
concretos. 3 Teniendo en cuenta un mismo sistema teórico, sería posible distinguir entre enunciados pertenecientes a diversos niveles de universalidad. Así, las diferentes ciencias serían caracterizadas por diferentes niveles internos de universalidad: "Los enunciados del nivel más alto son los axiomas, y de ellos pueden deducirse otros situados a niveles inferiores". Los enunciados empíricos de elevado nivel "tienen siempre el carácter de hipótesis con respecto a los enunciados — de nivel inferior- deductibles de ellos: pueden quedar falseados cuando se falsan esos enunciados menos universales". ${ }^{4}$

Según una epistemología de enfoque normativo, el método será, entonces, construido a partir del modo en que los enunciados supuestamente universales de dicha ciencia son reconocidos como válidos. La evolución científica sería, por tanto, dependiente de la progresiva capacidad de adaptación metodológica y resignificación epistémica del sistema teórico. A tal efecto, señalar la autonomía epistemológica de una ciencia implica, previamente, delimitar su sistema de fuentes a modo de cumplir la función de enunciados universales —o con elevado nivel de universalidad- y, especialmente, demonstrar la capacidad de dicha ciencia de producir sus propios aportes metodológicos.

Ante eso, innumerables dudas surgen al definir cómo solucionar los retos metodológicos en la labor editorial de una revista en un ámbito específico de conocimiento. Las ciencias exactas y las ciencias médicas se basan en la universalidad de los principios rectores que, incluso, asumen la condición de axiomas, para delimitar las posibilidades metodológicas y fortalecer la racionalidad instrumental. Sin embargo, ¿qué hacer cuando nos enfrentamos a diferentes metodologías posibles y, por supuesto, a diferentes racionalidades supuestas en la misma área? Las revistas jurídicas se enfrentan con el reto de contestar a tales preguntas y buscan ofrecer cierta "neutralidad metodológica" o, por expresar su propio perfil editorial, una orientación precisa sobre las opciones metodológicas que deberían guiar las investigaciones enviadas que aceptarán evaluar. Sin embargo, puede apuntarse otro problema: ¿es posible utilizar la racionalidad científico-instrumental en la ciencia jurídica de manera universal?

\footnotetext{
3 Popper, Karl, La lógica de la investigación científica, Madrid, Tecnos, 1962, p. 58.

${ }^{4}$ Popper, Karl, op. cit., p. 72.
} 


\section{Los límites de la racionalidad científico-instrumental en el derecho y la leyenda de su uso universal}

Una discusión clásica en la ciencia jurídica contemporánea, que no podremos recuperar aquí en ese formato de ensayo, es la distinción entre racionalidad normativa y teórica. En pocas palabras, la racionalidad normativa tendría la intención de ordenar su objeto para un propósito dado, mientras que la racionalidad teórica trataría de explicar las relaciones causales que justifican un objeto dado. En ambas racionalidades, la existencia de principios universalmente válidos no es un requisito fundamental, ya que el primero puede establecer libremente sus propios principios de ordenamiento, y el segundo se caracteriza por tener como propio principio de ordenamiento su capacidad para cuestionar cualesquiera principios de ordenamiento En ese sentido, las revistas jurídicas terminaron asumiendo, durante los últimos dos siglos, desde la creación y publicación continua de algunas de las más antiguas revistas jurídicas en Occidente, esa misma división elemental para, por un lado, establecer que una revista tiene un perfil "práctico", pragmático o normativo, es decir, en una palabra, dogmático; mientras que, por otro lado, una dada revista es de perfil teórico 0 , en una palabra, filosófico.

En las dos primeras décadas del siglo XXI se ha observado que las revistas jurídicas buscan el uso de la racionalidad científico-instrumental como una medida para la definición de qué investigación sería, de hecho, de alcance universal. La opción de publicar en inglés también ha sido una característica muy presente en esos casos, especialmente en revistas alemanas y en algunas italianas que han ganado un gran alcance mundial. Los índices internacionales de mayor alcance, como Scopus y Web of Science, también corroboran el hecho de que el inglés es el idioma lingüístico para transmitir investigaciones de supuesta validez universal.

En este momento surge una pregunta inevitable: ¿la investigación llevada a cabo por tales revistas es realmente universal o es una investigación que solo trata con objetos de "interés universal" basada en racionalidades normativas 0 teóricas? Cuando pensamos en el factor de impacto como el criterio con mayor grado de diseminación y la supuesta validad universal de una investigación, se puede señalar que las revistas con el mayor número de citas son revistas estadounidenses, pero su investigación también se centra en racionalidades 
normativas o teóricas. Además, las investigaciones publicadas en los Law Review estadounidenses, generalmente, analizan objetos de estudio de relevancia, principalmente para contextos locales o, cuando mucho, para el contexto nacional de Estados Unidos de América.

La inexistencia de supuestos epistemológicos con validad universal, como las Leyes de Newton para la física o las actualizaciones constantes del genoma humano para las ciencias médicas, por ejemplo, hacen que el uso de la racionalidad científico-instrumental se transfigure en una "leyenda" de la ciencia jurídica cuya aplicabilidad está restringida a objetos de estudio de interés universal y basados en supuestos epistemológicos aceptados por las tradiciones jurídicas más diferentes del mundo. En suma, superar el "mito" de la racionalidad científico-universal parece ser un primer paso para definir un perfil editorial que permita a una revista jurídica establecer criterios efectivos en el proceso de evaluación de las investigaciones recibidas.

\section{Algunas consideraciones finales basadas en experiencias de la RECHTD}

\section{y otras revistas}

Me permito, en esta fase concluyente de este ensayo, presentarles algunas experiencias que he tenido, tanto como editor-in-chief de la Revista de Estudos Constitucionais, Hermenêutica e Teoria do Direito (RECHTD) como consejero o miembro del comité de redacción de otras publicaciones jurídicas.

RECHTD es una revista exclusivamente electrónica mantenida por la Escuela Doctoral en Derecho de la Universidade do Vale do Rio dos Sinos (Unisinos), en Brasil. Creada a principios de 2009, reemplazó una revista impresa muy tradicional, la Estudos Jurídicos, que tenía un alcance limitado, aunque fue conocida y tradicional durante décadas en el universo académico brasileño. El hecho de que Estudos Jurídicos fuera solo una revista impresa la hizo, por supuesto, de alcance limitado. Por esta razón, RECHTD se creó con la intención de ingresar rápidamente a los principales índices internacionales y lograr el mayor alcance internacional. El prestigio de la Escuela Doctoral en Derecho, de la Unisinos, en el sistema de evaluación universitaria de Brasil hizo que la transición a RECHTD fuera un proceso muy rápido y aceptado por la comunidad académica nacional. En 2013, cuando asumí el papel de editor, recibíamos alrededor de 60 envíos de artículos por año, y los autores eran 
prácticamente todos brasileños. En 2016, cuando entramos en la base Scopus, el número ya era de 100 envíos por año, y casi todos provenían de Brasil. El mismo año ingresamos en otros índices internacionales importantes, como Dialnet, por ejemplo. Al año siguiente, 2017, recibimos 280 envíos. En 2018 llegamos a casi 400 envíos. Finalmente, 2019 terminó con la superación de la marca de más de 400 envíos recibidos en un año.

El informe estadístico anterior es relevante para la comprensión de los problemas editoriales que enfrentamos. En 2013, la evaluación de los envíos fue algo que, a través de una revisión por sistema doble ciego, podría hacerse fácilmente con cerca de 50 revisores. Los criterios editoriales también han sido fácilmente cumplidos por los autores, ya que casi todos eran nacionales. Resulta que, a partir de 2018, nuevos retos han surgido a partir de una cuestión que es central, en mi opinión: ¿cómo evaluar los envíos de Ucrania, China, Rusia, Italia, Indonesia, Inglaterra, Uruguay, entre otros países, con los mismos criterios editoriales y aplicando la existencia de metodologías que pueden ser universalmente compartibles? De los más de 400 envíos en 2019, aproximadamente 80 provinieron de 16 países diferentes.

Aunque es un tema de intensa discusión en el ámbito del consejo editorial de RECHTD y del colegiado de profesores de la Escuela de Doctorado que mantiene la revista, surgieron dos puntos relevantes: (1) evaluar solo la investigación que desarrolla un debate teóricamente de acceso universal, es decir, sobre temas universales y que se basan en doctrinas no restrictas a una realidad nacional limitada; (2) vincular los envíos, en el proceso de revisión por doble ciego, a los evaluadores que tienen conocimiento específico de las realidades locales, especialmente en la tradición jurídica de origen del artículo, y que pueden examinar profundamente las opciones metodológicas de los autores de los artículos. Como resultado, aunque contando con casi 250 revisores de más de 20 países, algunos artículos se analizan durante más de 12 meses, toda vez que para ciertos temas hay muy pocos revisores disponibles; además, con el objetivo de mantener a los evaluadores sin "sobrecargas", no se solicitan más de dos evaluaciones por año para cada colaborador. Por tanto, nuestra experiencia en RECHTD ha señalado la necesidad de una gradación cada vez más limitada de opciones metodológicas por parte de los autores. 
Vale la pena mencionar otras dos experiencias editoriales interesantes en las que trabajo como consejero editorial. La revista Interesse Público, bimestral, en formato impreso y electrónico, existe desde hace más de dos décadas, y actualmente es editada por la editoral Fórum, con amplio alcance entre el público brasileño. La revista Interesse Público está estrechamente vinculada con la dogmática jurídica y, en consecuencia, con el uso de la racionalidad instrumental ha sido de gran utilidad para definir qué envíos examinar o no. Muchas investigaciones que tratan de políticas públicas o temas de alta relevancia práctica dentro del alcance del derecho público brasileño pueden examinarse mediante un sistema doble ciego de revisión con cierta velocidad y objetividad, ya que tales artículos adoptan supuestos teóricos universalmente válidos. Como evaluador ciego, es común recibir investigaciones que explican metodológicamente de manera clara cómo se produjo el uso de la racionalidad instrumental en ese estudio en particular, para demostrar que la hipótesis teórica puede, de hecho, ser confirmada.

Una última experiencia que me parece interesante informar es la Revista Síntese de Direito Empresarial, bimestral, en formato impreso y electrónico, en la que he colaborado como consejero desde su creación, hace unos 15 años. Con perfil editorial bien delimitado y objetivo, permite que los autores dispongan de una gama más limitada de opciones metodológicas, por lo que el número de envíos también se encuentra dentro de márgenes factibles para que su editor la pueda gestionar más fácilmente. El hecho de ser una revista bimestral, centrada en el derecho comercial, permite incluso que ciertos números se publiquen con una alta articulación temática entre los textos, una articulación incluso difícil de hallarse en libros organizados con diferentes autores.

Conclusivamente, entiendo que el perfil editorial de una revista jurídica será decisivo en relación con cuáles metodologías y, como antecedente lógico, a cuáles racionalidades podrán guiarse los artículos que se publicarán en sus números. Sin embargo, superar la "leyenda" de la aplicabilidad universal de la racionalidad científico-instrumental en la ciencia jurídica me parece importante en el sentido de permitir que investigaciones de diferentes tradiciones jurídicas circulen entre diferentes países. No olvidemos, como señaló Horkheimer, que un rigor metodológico estéril meramente instrumental puede ocultar las 
pretensiones hegemónicas en las ciencias, reduciendo el espacio de la crítica y convirtiendo la razón en un instrumento de poder.

La razón debe entenderse como un concepto multidimensional en constante construcción, abierto a varias concepciones posibles, regidas filosóficamente por la búsqueda universal de la emancipación intelectual del ser humano y, sociológicamente, por la construcción de intersubjetividades basadas en procesos racionales compatibles entre sí. El aferramiento a racionalidades estrictas, especialmente en ciencia jurídica, conduce al eterno peligro de las ideologías cientificistas desprovistas de un sentido crítico o una base humanista aunque de minimus.

\section{Referencias}

Bunge, Mario, La ciencia: su método y su filosofía, Buenos Aires, Sudamericana, 1995.

Horkheimer, Max, Éclipse de la raison, Paris, Payot, 1974.

Popper, Karl, La lógica de la investigación científica, Madrid, Tecnos, 1962. 